\title{
On higher-power moments of $\Delta(x)$ (II)
}

\author{
by
}

\author{
Wenguang Zhai (Jinan)
}

\section{Introduction and main results}

1.1. Notations. Throughout this paper, let $d(n)$ denote the Dirichlet divisor function, $r(n)$ the number of ways $n$ can be written as $n=x^{2}+y^{2}$ for $x, y \in \mathbb{Z}$, and $a(n)$ the Fourier coefficients of a holomorphic cusp form of weight $\kappa=2 n \geq 12$ for the full modular group, $\widetilde{a}(n):=a(n) n^{-\kappa / 2+1 / 2}$. For short, we use $d, r, a, \tilde{a}$ to denote these functions, respectively. $\zeta(s)$ denotes the Riemann zeta-function.

Suppose $x, t>0$. Define

$$
\begin{aligned}
\Delta(x) & :=\sum_{n \leq x} d(n)-x \log x-(2 \gamma-1) x, \\
P(x) & :=\sum_{n \leq x} r(n)-\pi x, \\
A(x) & :=\sum_{n \leq x} a(n), \\
E(t) & :=\int_{0}^{t}|\zeta(1 / 2+i u)|^{2} d u-t \log (t / 2 \pi)-(2 \gamma-1) t .
\end{aligned}
$$

Suppose $f: \mathbb{N} \rightarrow \mathbb{R}$ is any function such that $f(n) \ll n^{\varepsilon}, k \geq 2$ is a fixed integer. Define

$$
s_{k ; l}(f):=\sum_{\sqrt{n_{1}}+\ldots+\sqrt{n_{l}}=\sqrt{n_{l+1}}+\ldots+\sqrt{n_{k}}} \frac{f\left(n_{1}\right) \ldots f\left(n_{k}\right)}{\left(n_{1} \ldots n_{k}\right)^{3 / 4}} \quad(1 \leq l<k),
$$

2000 Mathematics Subject Classification: 11N37, 11M06.

Key words and phrases: higher-power moment, Dirichlet divisor problem, asymptotic formula.

This work is supported by the National Natural Science Foundation of China (Grant No. 10301018). 


$$
B_{k}(f):=\sum_{l=1}^{k-1}\left(\begin{array}{c}
k-1 \\
l
\end{array}\right) s_{k ; l}(f) \cos \frac{\pi(k-2 l)}{4} .
$$

We shall use $s_{k ; l}(f)$ to denote both the series (1.5) and its value. We will prove the convergence of $s_{k ; l}(f)$ in Section 3.

Suppose $A_{0}>2$ is a real number. Define

$$
\begin{aligned}
K_{0} & :=\min \left\{n \in \mathbb{N}: n \geq A_{0}, 2 \mid n\right\}, \quad b(k):=2^{k-2}+(k-6) / 4, \\
\sigma\left(k, A_{0}\right) & := \begin{cases}1 / 4 & \text { if } k-1<A_{0} / 2, \\
\frac{A_{0}-k}{2\left(A_{0}-2\right)} & \text { if } A_{0} / 2+1 \leq k<A_{0},\end{cases} \\
\delta_{1}\left(k, A_{0}\right) & :=\frac{\sigma\left(k, A_{0}\right)}{2 b\left(K_{0}\right)}, \quad \delta_{2}\left(k, A_{0}\right):=\frac{\sigma\left(k, A_{0}\right)}{2 b(k)+2 \sigma\left(k, A_{0}\right)} .
\end{aligned}
$$

$\mathbb{N}$ denotes the set of all natural numbers; $\varepsilon$ always denotes a sufficiently small positive constant which may be different at different places. We will use the inequality $d(n) \ll n^{\varepsilon}$ freely. $\mathrm{SC}\left(\sum\right)$ denotes the summation condition of the $\operatorname{sum} \sum ; \mu(n)$ is the Möbius function.

1.2. Introduction. In this paper we shall study the higher-power moments of $\Delta(x), P(x), A(x)$ and $E(t)$.

We begin with the Dirichlet divisor problem. Dirichlet first proved that $\Delta(x)=O\left(x^{1 / 2}\right)$. The exponent $1 / 2$ was improved by many authors. The latest result reads

$$
\Delta(x) \ll x^{23 / 73}(\log x)^{315 / 146},
$$

which can be found in Huxley [6] (see also "Note added in proof"). It is conjectured that

$$
\Delta(x)=O\left(x^{1 / 4+\varepsilon}\right),
$$

which is supported by the classical mean-square result

$$
\int_{1}^{T} \Delta^{2}(x) d x=\frac{(\zeta(3 / 2))^{4}}{6 \pi^{2} \zeta(3)} T^{3 / 2}+O\left(T \log ^{5} T\right)
$$

proved by Tong [17] and the upper bound estimate

$$
\int_{1}^{T}|\Delta(x)|^{A_{0}} d x \ll T^{1+A_{0} / 4+\varepsilon}
$$

where $A_{0}>2$ is a fixed real number. The estimate of type (1.10) can be found in Ivić [7, Thm. 13.9] with $A_{0}=35 / 4$ and Heath-Brown [5] with $A_{0}=28 / 3$. On the other hand, Voronoï [19] proved that

$$
\int_{1}^{T} \Delta(x) d x=T / 4+O\left(T^{3 / 4}\right)
$$


which in conjunction with (1.9) shows that $\Delta(x)$ has a lot of sign changes and cancellations between the positive and negative portions.

Tsang [18] first studied the third- and fourth-power moments of $\Delta(x)$. He proved that (with notations of Section 1.1)

$$
\begin{aligned}
& \int_{1}^{T} \Delta^{3}(x) d x=\frac{3 s_{3 ; 1}(d)}{28 \pi^{3}} T^{7 / 4}+O\left(T^{7 / 4-1 / 14+\varepsilon}\right), \\
& \int_{1}^{T} \Delta^{4}(x) d x=\frac{3 s_{4 ; 2}(d)}{64 \pi^{4}} T^{2}+O\left(T^{2-1 / 23+\varepsilon}\right) .
\end{aligned}
$$

Heath-Brown [5] proved that for $k=3, \ldots, 9$ the limit

$$
\lim _{T \rightarrow \infty} T^{-1-k / 4} \int_{1}^{T} \Delta(x)^{k} d x
$$

exists.

In [20] the author improved Tsang's method and proved that

$$
\begin{aligned}
& \int_{1}^{T} \Delta^{3}(x) d x=\frac{3 s_{3 ; 1}(d)}{28 \pi^{3}} T^{7 / 4}+O\left(T^{3 / 2+\varepsilon}\right), \\
& \int_{1}^{T} \Delta^{4}(x) d x=\frac{3 s_{4 ; 2}(d)}{64 \pi^{4}} T^{2}+O\left(T^{2-2 / 41}\right), \\
& \int_{1}^{T} \Delta^{5}(x) d x=\frac{5\left(2 s_{5 ; 2}(d)-s_{5 ; 1}(d)\right)}{288 \pi^{5}} T^{9 / 4}+O\left(T^{9 / 4-5 / 816}\right) .
\end{aligned}
$$

But the argument of [20] fails for $k \geq 6$.

1.3. New results on higher-power moments of $\Delta(x)$. In this paper we shall use a different approach to study the higher-power moments of $\Delta(x)$. This leads to the asymptotic formulas for the integral $\int_{1}^{T} \Delta^{k}(x) d x$ for $3 \leq$ $k \leq 9$. Furthermore, if the estimate (1.8) is true, then our approach can give the asymptotic formulas for $\int_{1}^{T} \Delta^{k}(x) d x$ for any $k \geq 10$.

TheOrem 1. Let $A_{0}>9$ be a real number such that (1.10) holds. Then for any integer $3 \leq k<A_{0}$, we have the asymptotic formula

$$
\int_{1}^{T} \Delta^{k}(x) d x=\frac{B_{k}(d)}{(1+k / 4) 2^{3 k / 2-1} \pi^{k}} T^{1+k / 4}+O\left(T^{1+k / 4-\delta_{1}\left(k, A_{0}\right)+\varepsilon}\right) .
$$

Remark 1.1. From Ivić's argument [7, Thm. 13.9], we know that the value of $A_{0}$ for which (1.10) holds depends on the large-value estimate and the upper bound estimate of $\Delta(x)$. If we insert the estimate (1.7) into the argument of Ivić, we find that (1.10) holds with $A_{0}=184 / 19$. Hence for $k \in\{3,4,5,6,7,8,9\}$, we get the asymptotic formula (1.17). Moreover, if the 
estimate $\Delta(x) \ll x^{5 / 16-\delta}$ holds for some small $\delta>0$, then the asymptotic formula (1.17) holds for $k=10$.

REMARK 1.2. For $k \geq 10$, Theorem 1 is only a conditional result. However, it tells us that for any $k \geq 10$, the main term in the asymptotic formula for $\int_{1}^{T} \Delta^{k}(x) d x$ (if it exists) must have the form stated in (1.17).

REMARK 1.3. We can state the following three conjectures about $\Delta(x)$ :

Conjecture 1. The estimate (1.8) is true.

Conjecture 2. The estimate (1.10) is true for any $A_{0}>2$.

Conjecture 3 . For any fixed $k \geq 3$, there exists a constant $\delta_{k}>0$ such that the following asymptotic formula holds:

$$
\int_{1}^{T} \Delta^{k}(x) d x=\frac{B_{k}(d)}{(1+k / 4) 2^{3 k / 2-1} \pi^{k}} T^{1+k / 4}+O\left(T^{1+k / 4-\delta_{k}+\varepsilon}\right) .
$$

It is well known that Conjectures 1 and 2 are equivalent. From Theorem 1 we know that actually the three conjectures are equivalent. It is easy to deduce Conjecture 2 from Conjecture 3. To deduce Conjecture 3 from Conjecture 2, we take $A_{0}=2(k-1)$ and $\delta_{k}=\delta_{1}(k, 2(k-1))$.

Remark 1.4. From (1.11) we know that the integral $\int_{1}^{T} \Delta(x) d x$ has many cancellations from the positive and negative portions of $\Delta(x)$. However, from (1.12) Tsang [18] observed that this is not so for $\int_{1}^{T} \Delta^{3}(x) d x$. From Theorem 1 we know that this is also not so for $\int_{1}^{T} \Delta^{k}(x) d x(k=5,7,9)$ since numerical computation tells $B_{k}(d)>0$ for $k=5,7,9$. Maybe $B_{k}(d)>0$ holds for any odd $k \geq 3$.

The constant $\delta_{1}\left(k, A_{0}\right)$ is small for $k$ small. If we combine Ivić's argument with the proof of Theorem 1, we get the following Theorem 2 for $3 \leq k \leq 9$. Note that the results for $k=3,4$ are weaker than those of [20]. Theorem 2 for $k=5$ improves (1.16).

TheOREM 2. For $3 \leq k \leq 9$, the asymptotic formula (1.17) holds with $\delta_{1}\left(k, A_{0}\right)$ replaced by $\delta_{2}(k, 184 / 19)$.

In particular, for $k=5,6,7,8,9$, we have

$$
\begin{aligned}
\int_{1}^{T} \Delta^{5}(x) d x= & \frac{5\left(2 s_{5 ; 2}(d)-s_{5 ; 1}(d)\right)}{288 \pi^{5}} T^{9 / 4}+O\left(T^{9 / 4-1 / 64+\varepsilon}\right) \\
\int_{1}^{T} \Delta^{6}(x) d x= & \frac{5 s_{6 ; 3}(d)-3 s_{6 ; 1}(d)}{320 \pi^{6}} T^{5 / 2}+O\left(T^{5 / 2-35 / 4742+\varepsilon}\right) \\
\int_{1}^{T} \Delta^{7}(x) d x= & \frac{7\left(5 s_{7 ; 3}(d)-3 s_{7 ; 2}(d)-s_{7 ; 1}(d)\right)}{2816 \pi^{7}} T^{11 / 4} \\
& +O\left(T^{11 / 4-17 / 6312+\varepsilon}\right)
\end{aligned}
$$




$$
\begin{aligned}
\int_{1}^{T} \Delta^{8}(x) d x= & \frac{7\left(5 s_{8 ; 4}(d)-4 s_{8 ; 2}(d)\right)}{6144 \pi^{8}} T^{3}+O\left(T^{3-8 / 9433+\varepsilon}\right) \\
\int_{1}^{T} \Delta^{9}(x) d x= & \frac{3\left(3 s_{9 ; 1}(d)-12 s_{9 ; 2}(d)-28 s_{9 ; 3}(d)+42 s_{9 ; 4}(d)\right)}{26624 \pi^{9}} T^{13 / 4} \\
& +O\left(T^{13 / 4-13 / 75216+\varepsilon}\right) .
\end{aligned}
$$

1.4. Higher-power moments of $P(x), A(x)$ and $E(t)$. The method of proving Theorems 1 and 2 can also be applied to study the higher-power moments of $P(x), A(x)$ and $E(t)$.

The conjectured bound of $P(x)$ is

$$
P(x)=O\left(x^{1 / 4+\varepsilon}\right),
$$

which is supported by

$$
\int_{2}^{T} P^{2}(x) d x=\left(\frac{1}{3 \pi^{2}} \sum_{n=1}^{\infty} r^{2}(n) n^{-3 / 2}\right) T^{3 / 2}+O\left(T \log ^{2} T\right)
$$

proved by Kátai [14]. Tsang [18] also studied the third- and fourth-power moments of $P(x)$. His results were improved by the present author [20]. An asymptotic formula for the fifth-power moment of $P(x)$ was also obtained in [20], which is further improved by the following (for $k=5$ ):

THEOREM 3. Let $A_{0}>9$ be a real number such that

$$
\int_{1}^{T}|P(x)|^{A_{0}} d x \ll T^{1+A_{0} / 4+\varepsilon} .
$$

Then for any integer $3 \leq k<A_{0}$, the following asymptotic formula holds:

$$
\int_{1}^{T} P^{k}(x) d x=\frac{(-1)^{k} B_{k}(r)}{(1+k / 4) 2^{k-1} \pi^{k}} T^{1+k / 4}+O\left(T^{1+k / 4-\delta_{1}\left(k, A_{0}\right)+\varepsilon}\right) .
$$

In particular, for $3 \leq k \leq 9$, (1.26) holds with $\delta_{1}\left(k, A_{0}\right)$ replaced by $\delta_{2}(k, 184 / 19)$.

REMARK 1.5. Ivić [7, Thm. 13.12] proved that the estimate (1.25) holds for $A_{0}=35 / 4$. If we insert the estimate $P(x)=O\left(x^{23 / 73+\varepsilon}\right)$ (see Huxley [6]) into his argument, we find that (1.25) holds for $A_{0}=184 / 19$.

It is well known that $A(x)$ has no main term and $A(x) \ll x^{\kappa / 2-1 / 6+\varepsilon}$. From Deligne [4], we have $|\widetilde{a}(n)| \leq d(n)$.

The conjectured bound of $A(x)$ is $A(x) \ll x^{\kappa / 2-1 / 4+\varepsilon}$. Ivic [9] proved that

$$
\int_{1}^{T} A^{2}(x) d x=B_{2} T^{\kappa+1 / 2}+O\left(T^{\kappa} \log ^{5} T\right)
$$


where

$$
B_{2}=\frac{1}{4 \kappa+2} \sum_{n=1}^{\infty} a^{2}(n) n^{-\kappa-1 / 2} .
$$

Ivić [9] also proved that

$$
\int_{1}^{T}|A(x)|^{A_{0}} d x \ll T^{1+A_{0}(2 \kappa-1) / 4+\varepsilon}
$$

for $A_{0}=8$. Cai [3] studied the third- and fourth-power moments of $A(x)$. His results were improved in [20], where an asymptotic formula for the fifthpower moment of $A(x)$ was also obtained, which is further improved by the case $k=5$ of the following:

TheOREm 4. Let $A_{0} \geq 8$ be a real number such that (1.28) is true. Then for any $3 \leq k<A_{0}$, we have the asymptotic formula

$$
\begin{aligned}
\int_{1}^{T} A^{k}(x) d x= & \frac{B_{k}(\widetilde{a})}{\left(1+\frac{k(2 \kappa-1)}{4}\right) 2^{3 k / 2-1} \pi^{k}} T^{1+\frac{k(2 \kappa-1)}{4}} \\
& +O\left(T^{1+\frac{k(2 \kappa-1)}{4}-\delta_{1}\left(k, A_{0}\right)+\varepsilon}\right) .
\end{aligned}
$$

In particular, for $3 \leq k \leq 7$, (1.29) holds with $\delta_{1}\left(k, A_{0}\right)$ replaced by $\delta_{2}(k, 8)$.

Many results for $E(t)$ parallel to those for $\Delta(x)$ have been obtained; see Ivić [8] for a survey. The conjectured bound for $E(t)$ is $E(t) \ll t^{1 / 4+\varepsilon}$, which is supported by

$$
\int_{2}^{T} E^{2}(t) d t=\frac{2 \zeta^{4}(3 / 2)}{3 \zeta(3) \sqrt{2 \pi}} T^{3 / 2}+O\left(T \log ^{5} T\right),
$$

proved by Meurman [15]. It has been proved (see Huxley [6]) that

$$
E(t) \ll t^{72 / 227}(\log t)^{629 / 227}, \quad t>2 .
$$

Ivić $[7$, Thm. 15.7] proved that

$$
\int_{1}^{T}|E(t)|^{A_{0}} d t \ll T^{1+A_{0} / 4+\varepsilon}
$$

for $A_{0}=35 / 4$. Inserting (1.31) into Ivić's argument, we find that (1.32) is true for $A_{0}=576 / 61$.

Tsang [18] studied the third- and fourth-power moment of $E(t)$ by using the Atkinson formula [1]. His results were further improved by Ivić [10] in a different way. The author [20] obtained new results on the third- and fourthpower moments of $E(t)$. An asymptotic formula for the fifth-power moment 
of $E(t)$ was also obtained in [20], which is further improved by the case $k=5$ of the following:

THEOREM 5. Let $A_{0}>9$ be a real number such that the estimates (1.10) and (1.32) hold. Then for any $3 \leq k<A_{0}$, we have the asymptotic formula

$$
\int_{1}^{T} E^{k}(t) d t=\frac{B_{k}(d)}{(1+k / 4) 2^{3 k / 4-1} \pi^{k / 4}} T^{1+k / 4}+O\left(T^{1+k / 4-\delta_{1}\left(k, A_{0}\right)+\varepsilon}\right) .
$$

In particular, for $3 \leq k \leq 9$, (1.33) holds with $\delta_{1}\left(k, A_{0}\right)$ replaced by $\delta_{2}(k, 576 / 61)$.

Acknowledgements. The author deeply thanks the referee for his warm and valuable comments. The author also thanks Prof. Isao Wakabayashi for helpful discussions, through which the proof of Lemma 3.1 was further improved.

2. Some preliminary lemmas. We need the following lemmas.

LEMMA 2.1. The square roots of squarefree numbers are linearly independent over the integers.

Proof. This is a classical result of Besicovitch [2].

LEMma 2.2. Suppose $k \geq 3$ and $\left(i_{1}, \ldots, i_{k-1}\right) \in\{0,1\}^{k-1}$ are such that

$$
\sqrt{n_{1}}+(-1)^{i_{1}} \sqrt{n_{2}}+(-1)^{i_{2}} \sqrt{n_{3}}+\ldots+(-1)^{i_{k-1}} \sqrt{n_{k}} \neq 0
$$

Then

$$
\begin{aligned}
\mid \sqrt{n_{1}}+(-1)^{i_{1}} \sqrt{n_{2}}+(-1)^{i_{2}} \sqrt{n_{3}}+\ldots & +(-1)^{i_{k-1}} \sqrt{n_{k}} \mid \\
& \gg \max \left(n_{1}, \ldots, n_{k}\right)^{-\left(2^{k-2}-2^{-1}\right)} .
\end{aligned}
$$

Proof. The cases $k=3,4$ are Lemmas 1 and 2 of Tsang [18], respectively. The proof for the general case is the same as the proof of Lemma 1 of [18]. We note that Heath-Brown [5] stated a similar result for $k$ even.

Lemma 2.3. Suppose $A, B \in \mathbb{R}, A \neq 0$. Then

$$
\int_{T}^{2 T} \cos (A \sqrt{t}+B) d t \ll T^{1 / 2}|A|^{-1} .
$$

Lemma 2.4. Suppose $k \geq 3,\left(i_{1}, \ldots, i_{k-1}\right) \in\{0,1\}^{k-1},\left(i_{1}, \ldots, i_{k-1}\right) \neq$ $(0, \ldots, 0), N_{1}, \ldots, N_{k}>1,0<\Delta \ll E^{1 / 2}, E=\max \left(N_{1}, \ldots, N_{k}\right)$. Let

$$
\mathcal{A}=\mathcal{A}\left(N_{1}, \ldots, N_{k} ; i_{1}, \ldots, i_{k-1} ; \Delta\right)
$$

denote the number of solutions of the inequality

$$
\left|\sqrt{n_{1}}+(-1)^{i_{1}} \sqrt{n_{2}}+(-1)^{i_{2}} \sqrt{n_{3}}+\ldots+(-1)^{i_{k-1}} \sqrt{n_{k}}\right|<\Delta
$$


with $N_{j}<n_{j} \leq 2 N_{j}, 1 \leq j \leq k$. Then

$$
\mathcal{A} \ll \Delta E^{-1 / 2} N_{1} \ldots N_{k}+E^{-1} N_{1} \ldots N_{k} .
$$

Proof. Without loss of generality, suppose $E=N_{k}$. If $\left(n_{1}, \ldots, n_{k}\right)$ satisfies (2.1), then

$$
\sqrt{n_{1}}+(-1)^{i_{1}} \sqrt{n_{2}}+(-1)^{i_{2}} \sqrt{n_{3}}+\ldots+(-1)^{i_{k-2}} \sqrt{n_{k-1}}=(-1)^{i_{k-1}+1} \sqrt{n_{k}}+\theta \Delta
$$

for some $|\theta|<1$, whence we get

$$
\left(\sqrt{n_{1}}+(-1)^{i_{1}} \sqrt{n_{2}}+(-1)^{i_{2}} \sqrt{n_{3}}+\ldots+(-1)^{i_{k-2}} \sqrt{n_{k-1}}\right)^{2}=n_{k}+O\left(\Delta N_{k}^{1 / 2}\right) .
$$

Hence for fixed $\left(n_{1}, \ldots, n_{k-1}\right)$, the number of $n_{k}$ is $\ll 1+\Delta N_{k}^{1 / 2}$ and thus

$$
\mathcal{A} \ll \Delta N_{k}^{1 / 2} N_{1} \ldots N_{k-1}+N_{1} \ldots N_{k-1} .
$$

3. On the series $s_{k ; l}(d)$. Suppose $y>1$ is a large parameter, and define

$$
s_{k ; l}(d ; y):=\sum_{\substack{\sqrt{n_{1}}+\ldots+\sqrt{n_{l}}=\sqrt{n_{l+1}}+\ldots+\sqrt{n_{k}} \\ n_{1}, \ldots, n_{k} \leq y}} \frac{d\left(n_{1}\right) \ldots d\left(n_{k}\right)}{\left(n_{1} \ldots n_{k}\right)^{3 / 4}}, \quad 1 \leq l<k .
$$

We shall prove

Lemma 3.1. We have

$$
\left|s_{k ; l}(d)-s_{k ; l}(d ; y)\right| \ll y^{-1 / 2+\varepsilon}, \quad 1 \leq l<k .
$$

REMARK. Lemma 3.1 is still true if the divisor function $d$ is replaced by any function $f: \mathbb{N} \rightarrow \mathbb{R}$ with $f(n) \ll n^{\varepsilon}$.

Proof. We shall prove Lemma 3.1 by induction in $k$. The case $k=2$ is easy. The case $k=3$ is contained in [18, p. 70]. Later we suppose $k \geq 4$. Since $s_{k ; l}(d)=s_{k ; k-l}(d)$, we suppose $l \leq k / 2$.

By symmetry, we get

$$
\begin{aligned}
\left|s_{k ; l}(d)-s_{k ; l}(d ; y)\right| & \ll \sum_{\substack{\sqrt{n_{1}}+\ldots+\sqrt{n_{l}}=\sqrt{n_{l+1}}+\ldots+\sqrt{n_{k}} \\
n_{1}>y}} \frac{d\left(n_{1}\right) \ldots d\left(n_{k}\right)}{\left(n_{1} \ldots n_{k}\right)^{3 / 4}} \\
& \ll U_{1}(d ; y)+U_{2}(d ; y),
\end{aligned}
$$

say, where

$$
\begin{aligned}
& U_{1}(d ; y):=\sum_{j=l+1}^{k} \sum_{\substack{\sqrt{n_{1}}+\ldots+\sqrt{n_{l}}=\sqrt{n_{l+1}}+\ldots+\sqrt{n_{k}} \\
n_{1}=n_{j}>y}} \frac{d\left(n_{1}\right) \ldots d\left(n_{k}\right)}{\left(n_{1} \ldots n_{k}\right)^{3 / 4}}, \\
& U_{2}(d ; y):=\sum_{\substack{n_{n_{1}}+\ldots+\sqrt{n_{l}}=\sqrt{n_{l+1}}+\ldots+\sqrt{n_{k}} \\
n_{1}>y, n_{1} \neq n_{j}, l+1 \leq j \leq k}} \frac{d\left(n_{1}\right) \ldots d\left(n_{k}\right)}{\left(n_{1} \ldots n_{k}\right)^{3 / 4}} .
\end{aligned}
$$


If $l=1$, then obviously $U_{1}(d ; y)=0$. If $l>1$, then by induction we get

$$
U_{1}(d ; y) \ll \sum_{n>y} \frac{d^{2}(n)}{n^{3 / 2}} s_{k-2 ; l-1}(d) \ll y^{-1 / 2+\varepsilon} .
$$

Now we estimate $U_{2}(d ; y)$. Let $I=\{1, \ldots, l\}, J=\{l+1, \ldots, k\}$. Suppose $\left(n_{1}, \ldots, n_{k}\right) \in \mathbb{N}^{k}$ are such that

$$
\text { (*) } \quad \sqrt{n_{1}}+\ldots+\sqrt{n_{l}}=\sqrt{n_{l+1}}+\ldots+\sqrt{n_{k}}, \quad n_{1} \neq n_{j}, \quad l+1 \leq j \leq k .
$$

Then there exist two sets $I_{0} \subset I, J_{0} \subset J$ with the following properties:

(1) $1 \in I_{0}$;

(2) $\sum_{i \in I_{0}} \sqrt{n_{i}}=\sum_{j \in J_{0}} \sqrt{n_{j}}$;

(3) For any real subset $I_{0}^{\prime} \subset I_{0}, J_{0}^{\prime} \subset J_{0}$, we have

$$
\sum_{i \in I_{0}^{\prime}} \sqrt{n_{i}} \neq \sum_{j \in J_{0}^{\prime}} \sqrt{n_{j}}
$$

If $\left(I_{0}, J_{0}\right)=(I, J)$, then we say $\left(n_{1}, \ldots, n_{k}\right)$ is a primitive $(k, l)$-point. Let $\mathbb{N}_{k ; l}$ denote the set of all points in $\mathbb{N}^{k}$ which satisfy $(*)$ and $\mathbb{N}_{k ; l}^{*}$ the set of all primitive $(k, l)$-points. Let $\mathcal{G}_{k ; l}$ denote the set of all possible pairs $\left(I_{0}, J_{0}\right)$ when $\left(n_{1}, \ldots, n_{k}\right)$ runs through $\mathbb{N}_{k ; l}$. Note that if $l=1$, then $\mathcal{G}_{k ; l}=\{(I, J)\}$.

Suppose $\left(I_{0}, J_{0}\right) \in \mathcal{G}_{k ; l}$. Let $l_{1}=\# I_{0}, l_{2}=l-l_{1}, k_{1}=\# I_{0}+\# J_{0}, k_{2}=$ $k-k_{1}$. From $(*)$, we know that $k_{1} \geq 3$. Define

$$
R_{1}^{\left(I_{0}, J_{0}\right)}(d ; y):=\sum_{\substack{\sqrt{n_{1}}+\ldots+\sqrt{n_{l_{1}}}=\sqrt{n_{l_{1}+1}}+\ldots+\sqrt{n_{k_{1}}} \\ n_{1}>y,\left(n_{1}, \ldots, n_{k_{1}}\right) \in \mathbb{N}_{k_{1}}^{*} ; l_{1}}} \frac{d\left(n_{1}\right) \ldots d\left(n_{k_{1}}\right)}{\left(n_{1} \ldots n_{k_{1}}\right)^{3 / 4}} .
$$

If $\left(I_{0}, J_{0}\right) \neq(I, J)$, then $l_{1}<l, k_{1}<k$ and we define

$$
R_{2}^{\left(I_{0}, J_{0}\right)}(d):=\sum_{\sqrt{m_{1}}+\ldots+\sqrt{m_{l_{2}}}=\sqrt{m_{l_{2}+1}}+\ldots+\sqrt{m_{k_{2}}}} \frac{d\left(m_{1}\right) \ldots d\left(m_{k_{2}}\right)}{\left(m_{1} \ldots m_{k_{2}}\right)^{3 / 4}} .
$$

By the induction assumption, $R_{2}^{\left(I_{0}, J_{0}\right)}(d) \ll 1$.

If $\left(n_{1}, \ldots, n_{k_{1}}\right) \in \mathbb{N}_{k_{1} ; l_{1}}^{*}$, then by Lemma 2.1 we have

$$
n_{j}=s_{j}^{2} h, \quad s_{1}+\ldots+s_{l_{1}}=s_{l_{1}+1}+\ldots+s_{k_{1}}, \quad \mu(h) \neq 0 .
$$

Now $n_{1}>y$ implies that there exists at least one $n_{j}\left(l_{1}+1 \leq j \leq j_{1}\right)$ such that $n_{j} \gg y$. We suppose $n_{k_{1}} \gg y$. So we have

$$
R_{1}^{\left(I_{0}, J_{0}\right)}(d ; y) \ll \sum_{h} \sum_{\substack{s_{1}+\ldots+s_{l_{1}}=s_{l_{1}+1}+\ldots+s_{k_{1}} \\ s_{1}^{2} h>y, s_{k_{1}}^{2} h \gg y}} \frac{d\left(s_{1}^{2} h\right) \ldots d\left(s_{k_{1}}^{2} h\right)}{h^{3 k_{1} / 4}\left(s_{1} \ldots s_{k_{1}}\right)^{3 / 2}}
$$




$$
\begin{aligned}
& \ll \sum_{h} \sum_{\substack{s_{1}+\ldots+s_{l_{1}}=s_{l_{1}+1}+\ldots+s_{k_{1}} \\
s_{1}^{2} h>y, s_{k_{1}}^{2} h \gg y}} \frac{d^{2}\left(s_{1}\right) \ldots d^{2}\left(s_{k_{1}}\right) d^{k_{1}}(h)}{h^{3 k_{1} / 4}\left(s_{1} \ldots s_{k_{1}}\right)^{3 / 2}} \\
& \ll \sum_{h} \frac{d^{k_{1}}(h)}{h^{3 k_{1} / 4}} \sum_{s_{1}>(y / h)^{1 / 2}} \frac{d^{2}\left(s_{1}\right)}{s_{1}^{3 / 2}} \sum_{s_{k_{1}} \gg(y / h)^{1 / 2}} \frac{d^{2}\left(s_{k_{1}}\right)}{s_{k_{1}}^{3 / 2}} \\
& \ll \sum_{h} \frac{d^{k_{1}}(h)}{h^{3 k_{1} / 4}}\left(\frac{y}{h}\right)^{-1 / 2+\varepsilon} \ll y^{-1 / 2+\varepsilon}
\end{aligned}
$$

if we notice $k_{1} \geq 3$.

If $\mathcal{G}_{k ; l}=(I, J)$, we have

$$
U_{2}(d ; y) \ll R_{1}^{(I, J)}(d ; y) \ll y^{-1 / 2+\varepsilon} .
$$

If $\mathcal{G}_{k ; l} \neq(I, J)$, we have

$$
\begin{aligned}
U_{2}(d ; y) & \ll R_{1}^{(I, J)}(d ; y)+\sum_{\substack{\left(I_{0}, J_{0}\right) \in \mathcal{G}_{k ; l} \\
\left(I_{0}, J_{0}\right) \neq(I, J)}} R_{1}^{\left(I_{0}, J_{0}\right)}(d ; y) R_{2}^{\left(I_{0}, J_{0}\right)}(d) \\
& \ll y^{-1 / 2+\varepsilon} .
\end{aligned}
$$

Now Lemma 3.1 follows from (3.1)-(3.4).

4. Proofs of Theorems 1 and 2. Suppose $T \geq 10$ is a real number. It suffices to evaluate the integral $\int_{T}^{2 T} \Delta^{k}(x) d x$. Suppose $y$ is a parameter such that $T^{\varepsilon}<y \leq T^{1 / 3}$. For any $T \leq x \leq 2 T$, define

$$
\begin{aligned}
& \mathcal{R}_{1}=\mathcal{R}_{1}(x, y):=(\sqrt{2} \pi)^{-1} x^{1 / 4} \sum_{n \leq y} \frac{d(n)}{n^{3 / 4}} \cos (4 \pi \sqrt{x n}-\pi / 4), \\
& \mathcal{R}_{2}=\mathcal{R}_{2}(x, y):=\Delta(x)-\mathcal{R}_{1} .
\end{aligned}
$$

We shall show that the higher-power moment of $\mathcal{R}_{2}$ is small and hence the integral $\int_{T}^{2 T} \Delta^{k}(x) d x$ can be well approximated by $\int_{T}^{2 T} \mathcal{R}_{1}^{k} d x$, which is easy to evaluate.

4.1. Evaluation of the integral $\int_{T}^{2 T} \mathcal{R}_{1}^{h} d x$. Suppose $h \geq 3$ is any fixed integer. By the elementary formula

$$
\begin{aligned}
& \cos a_{1} \ldots \cos a_{h} \\
& =\frac{1}{2^{h-1}} \sum_{\left(i_{1}, \ldots, i_{h-1}\right) \in\{0,1\}^{h-1}} \cos \left(a_{1}+(-1)^{i_{1}} a_{2}+(-1)^{i_{2}} a_{3}+\ldots+(-1)^{i_{h-1}} a_{h}\right),
\end{aligned}
$$


we have

$$
\begin{aligned}
\mathcal{R}_{1}^{h}= & (\sqrt{2} \pi)^{-h} x^{h / 4} \sum_{n_{1} \leq y} \ldots \sum_{n_{h} \leq y} \frac{d\left(n_{1}\right) \ldots d\left(n_{h}\right)}{\left(n_{1} \ldots n_{h}\right)^{3 / 4}} \prod_{j=1}^{h} \cos \left(4 \pi \sqrt{n_{j} x}-\pi / 4\right) \\
= & \frac{x^{h / 4}}{(\sqrt{2} \pi)^{h} 2^{h-1}} \sum_{\left(i_{1}, \ldots, i_{h-1}\right) \in\{0,1\}^{h-1}} \ldots \sum_{n_{1} \leq y} \frac{d\left(n_{1}\right) \ldots d\left(n_{h}\right)}{\left(n_{1} \ldots n_{h}\right)^{3 / 4}} \\
& \times \cos \left(4 \pi \sqrt{x} \alpha\left(n_{1}, \ldots, n_{h} ; i_{1}, \ldots, i_{h-1}\right)-\frac{\pi}{4} \beta\left(i_{1}, \ldots, i_{h-1}\right)\right),
\end{aligned}
$$

where

$$
\begin{gathered}
\alpha\left(n_{1}, \ldots, n_{h} ; i_{1}, \ldots, i_{h-1}\right) \\
:=\sqrt{n_{1}}+(-1)^{i_{1}} \sqrt{n_{2}}+(-1)^{i_{2}} \sqrt{n_{3}}+\ldots+(-1)^{i_{h-1}} \sqrt{n_{h}} \\
\beta\left(i_{1}, \ldots, i_{h-1}\right):=1+(-1)^{i_{1}}+(-1)^{i_{2}}+\ldots+(-1)^{i_{h-1}} .
\end{gathered}
$$

Thus we can write

$$
\mathcal{R}_{1}^{h}=\frac{1}{(\sqrt{2} \pi)^{h} 2^{h-1}}\left(S_{1}(x)+S_{2}(x)\right),
$$

where

$$
\begin{gathered}
S_{1}(x):=x^{h / 4} \sum_{\left(i_{1}, \ldots, i_{h-1}\right) \in\{0,1\}^{h-1}} \cos \left(-\frac{\pi \beta}{4}\right) \sum_{\substack{n_{j} \leq y, 1 \leq j \leq h \\
\alpha=0}} \frac{d\left(n_{1}\right) \ldots d\left(n_{h}\right)}{\left(n_{1} \ldots n_{h}\right)^{3 / 4}}, \\
S_{2}(x):=x^{h / 4} \sum_{\substack{\left(i_{1}, \ldots, i_{h-1}\right) \in\{0,1\}^{h-1} \\
n_{j} \leq y, 1 \leq j \leq h \\
\alpha \neq 0}} \frac{d\left(n_{1}\right) \ldots d\left(n_{h}\right)}{\left(n_{1} \ldots n_{h}\right)^{3 / 4}} \\
\times \cos (4 \pi \alpha \sqrt{x}-\pi \beta / 4), \\
\alpha:=\alpha\left(n_{1}, \ldots, n_{h} ; i_{1}, \ldots, i_{h-1}\right), \quad \beta:=\beta\left(i_{1}, \ldots, i_{h-1}\right) .
\end{gathered}
$$

First consider the contribution of $S_{1}(x)$. We have

$$
\begin{aligned}
& \int_{T}^{2 T} S_{1}(x) d x \\
= & \sum_{\left(i_{1}, \ldots, i_{h-1}\right) \in\{0,1\}^{h-1}} \cos \left(-\frac{\pi \beta}{4}\right) \sum_{\substack{n_{j} \leq y, 1 \leq j \leq h \\
\alpha=0}} \frac{d\left(n_{1}\right) \ldots d\left(n_{h}\right)}{\left(n_{1} \ldots n_{h}\right)^{3 / 4}} \int_{T}^{2 T} x^{h / 4} d x .
\end{aligned}
$$

It is easily seen that if $\alpha=0$, then $1 \in\left\{i_{1}, \ldots, i_{h-1}\right\}$. Let $l=i_{1}+\ldots+i_{h-1}$. Then

$$
\sum_{\substack{\alpha=0 \\ n_{j} \leq y, 1 \leq j \leq h}} \frac{d\left(n_{1}\right) \ldots d\left(n_{h}\right)}{\left(n_{1} \ldots n_{h}\right)^{3 / 4}}=s_{h ; l}(d ; y),
$$

where $s_{h ; l}(d ; y)$ was defined in the last section. 
By Lemma 3.1 we get

$$
\int_{T}^{2 T} S_{1}(x) d x=B_{h}^{*}(d) \int_{T}^{2 T} x^{h / 4} d x+O\left(T^{1+h / 4+\varepsilon} y^{-1 / 2}\right)
$$

where

$$
B_{h}^{*}(d):=\sum_{\left(i_{1}, \ldots, i_{h-1}\right) \in\{0,1\}^{h-1}} \cos \left(-\frac{\pi \beta}{4}\right) \sum_{\substack{\left(n_{1}, \ldots, n_{h}\right) \in \mathbb{N}^{h} \\ \alpha=0}} \frac{d\left(n_{1}\right) \ldots d\left(n_{h}\right)}{\left(n_{1} \ldots n_{h}\right)^{3 / 4}} .
$$

For any $\left(i_{1}, \ldots, i_{h-1}\right) \in\{0,1\}^{h-1} \backslash\{(0, \ldots, 0)\}$, let

$$
\begin{aligned}
S\left(d ; i_{1}, \ldots, i_{h-1}\right) & :=\sum_{\substack{\left(n_{1}, \ldots, n_{h}\right) \in \mathbb{N}^{h} \\
\alpha=0}} \frac{d\left(n_{1}\right) \ldots d\left(n_{h}\right)}{\left(n_{1} \ldots n_{h}\right)^{3 / 4}}, \\
l\left(i_{1}, \ldots, i_{h-1}\right) & :=i_{1}+\ldots+i_{h-1} .
\end{aligned}
$$

It is easily seen that if $l\left(i_{1}, \ldots, i_{h-1}\right)=l\left(i_{1}^{\prime}, \ldots, i_{h-1}^{\prime}\right)$ or $l\left(i_{1}, \ldots, i_{h-1}\right)+$ $l\left(i_{1}^{\prime}, \ldots, i_{h-1}^{\prime}\right)=h$, then

$$
S\left(d ; i_{1}, \ldots, i_{h-1}\right)=S\left(d ; i_{1}^{\prime}, \ldots, i_{h-1}^{\prime}\right)=s_{h ; l\left(i_{1}, \ldots, i_{h-1}\right)}(d) .
$$

From $(-1)^{i}=1-2 i(i=0,1)$ we also have

$$
\beta\left(i_{1}, \ldots, i_{h-1}\right)=h-2 l\left(i_{1}, \ldots, i_{h-1}\right) .
$$

So we get

$$
\begin{aligned}
B_{h}^{*}(d) & =\sum_{l=1}^{h-1} \sum_{l\left(i_{1}, \ldots, i_{h-1}\right)=l} \cos \left(-\frac{\pi \beta}{4}\right) S\left(d ; i_{1}, \ldots, i_{h-1}\right) \\
& =\sum_{l=1}^{h-1} s_{h ; l}(d) \cos \frac{\pi(h-2 l)}{4} \sum_{l\left(i_{1}, \ldots, i_{h-1}\right)=l} 1 \\
& =\sum_{l=1}^{h-1}\left(\begin{array}{c}
h-1 \\
l
\end{array}\right) s_{h ; l}(d) \cos \frac{\pi(h-2 l)}{4}=B_{h}(d) .
\end{aligned}
$$

Now we consider the contribution of $S_{2}(x)$. By Lemma 2.3 we get

$$
\begin{aligned}
& \int_{T}^{2 T} S_{2}(x) d x \\
& \ll T^{1 / 2+h / 4} \sum_{\substack{\left(i_{1}, \ldots, i_{h-1}\right) \in\{0,1\}^{h-1} \\
n_{j} \leq y, 1 \leq j \leq h \\
\alpha \neq 0}} \frac{d\left(n_{1}\right) \ldots d\left(n_{h}\right)}{\left(n_{1} \ldots n_{h}\right)^{3 / 4}|\alpha|} .
\end{aligned}
$$


It suffices to estimate the sum

$$
\Sigma\left(y ; i_{1}, \ldots, i_{h-1}\right)=\sum_{\substack{n_{j} \leq y, 1 \leq j \leq h \\ \alpha \neq 0}} \frac{d\left(n_{1}\right) \ldots d\left(n_{h}\right)}{\left(n_{1} \ldots n_{h}\right)^{3 / 4}|\alpha|}
$$

for fixed $\left(i_{1}, \ldots, i_{h-1}\right) \in\{0,1\}^{h-1}$. If $\left(i_{1}, \ldots, i_{h-1}\right)=(0, \ldots, 0)$, then

$$
\begin{aligned}
\Sigma(y ; 0, \ldots, 0) & \ll \sum_{n_{j} \leq y, 1 \leq j \leq h} \frac{d\left(n_{1}\right) \ldots d\left(n_{h}\right)}{\left(n_{1} \ldots n_{h}\right)^{3 / 4}\left(\sqrt{n_{1}}+\ldots+\sqrt{n_{h}}\right)} \\
& \ll \sum_{n_{j} \leq y, 1 \leq j \leq h} \frac{d\left(n_{1}\right) \ldots d\left(n_{h}\right)}{\left(n_{1} \ldots n_{h}\right)^{3 / 4+1 / 2 h}} \ll y^{(h-2) / 4} \log ^{h} y,
\end{aligned}
$$

where we used the estimates

$$
\sum_{n \leq u} d(n) \ll u \log u, \quad x_{1}+\ldots+x_{h} \gg\left(x_{1} \ldots x_{h}\right)^{1 / h} .
$$

For $\left(i_{1}, \ldots, i_{h-1}\right) \neq(0, \ldots, 0)$, by a splitting argument we deduce that there exist a collection of numbers $1<N_{1}, \ldots, N_{h}<y$ such that

$$
\Sigma\left(y ; i_{1}, \ldots, i_{h-1}\right) \ll \Sigma_{1}^{*} \log ^{h} y,
$$

where

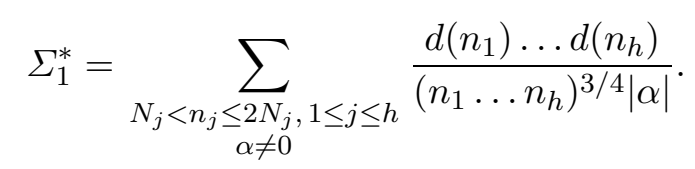

Without loss of generality, we suppose $N_{1} \leq \ldots \leq N_{h} \leq y$. By Lemma 2.2 we have $|\alpha| \gg N_{h}^{-\left(2^{h-2}-2^{-1}\right)}$. Then by a splitting argument and Lemma 2.4, for some $N_{h}^{-\left(2^{h-2}-2^{-1}\right)} \ll \Delta<y^{1 / 2}$ we get

$$
\begin{aligned}
\Sigma_{1}^{*} & \ll \frac{y^{\varepsilon}}{\left(N_{1} \ldots N_{h}\right)^{3 / 4} \Delta} \mathcal{A}\left(N_{1}, \ldots, N_{h} ; i_{1}, \ldots, i_{h-1} ; \Delta\right) \\
& \ll \frac{y^{\varepsilon}}{\left(N_{1} \ldots N_{h}\right)^{3 / 4} \Delta}\left(\Delta N_{h}^{1 / 2} N_{1} \ldots N_{h-1}+N_{1} \ldots N_{h-1}\right) \\
& \ll y^{\varepsilon}\left(\frac{\left(N_{1} \ldots N_{h-1}\right)^{1 / 4}}{N_{h}^{1 / 4}}+\frac{\left(N_{1} \ldots N_{h-1}\right)^{1 / 4}}{N_{h}^{3 / 4} \Delta}\right) \\
& \ll y^{\varepsilon}\left(N_{h}^{(h-2) / 4}+N_{h}^{b(h)}\right) \ll y^{b(h)+\varepsilon},
\end{aligned}
$$

where $b(h)$ was defined in Section 1.1. Thus we get

$$
\int_{T}^{2 T} S_{2}(x) d x \ll T^{1 / 2+h / 4+\varepsilon} y^{b(h)} .
$$


Hence from (4.1)-(4.6) we get

LEMMA 4.1. For any fixed $h \geq 3$, we have

$$
\begin{aligned}
\int_{T}^{2 T} \mathcal{R}_{1}^{h} d x= & \frac{B_{h}(d)}{(\sqrt{2} \pi)^{h} 2^{h-1}} \int_{T}^{2 T} x^{h / 4} d x \\
& +O\left(T^{1+h / 4+\varepsilon} y^{-1 / 2}+T^{1 / 2+h / 4+\varepsilon} y^{b(h)}\right) .
\end{aligned}
$$

4.2. Higher-power moments of $\mathcal{R}_{2}$. We first study the mean-square of $\mathcal{R}_{2}$. We begin with the truncated Voronoï formula [9, (2.25)]

$$
\begin{aligned}
\Delta(x)= & (\pi \sqrt{2})^{-1} x^{1 / 4} \sum_{n \leq N} \frac{d(n)}{n^{3 / 4}} \cos (4 \pi \sqrt{n x}-\pi / 4) \\
& +O\left(x^{1 / 2+\varepsilon} N^{-1 / 2}\right)
\end{aligned}
$$

where $1<N \ll x$. Taking $N=T$, we get

$$
\begin{aligned}
\mathcal{R}_{2} & =(\pi \sqrt{2})^{-1} x^{1 / 4} \sum_{y<n \leq T} \frac{d(n)}{n^{3 / 4}} \cos (4 \pi \sqrt{n x}-\pi / 4)+O\left(T^{\varepsilon}\right) \\
& \ll\left|x^{1 / 4} \sum_{y<n \leq T} \frac{d(n)}{n^{3 / 4}} e(2 \sqrt{n x})\right|+T^{\varepsilon},
\end{aligned}
$$

which implies

$$
\begin{aligned}
& \int_{T}^{2 T} \mathcal{R}_{2}^{2} d x \ll T^{1+\varepsilon}+\int_{T}^{2 T}\left|x^{1 / 4} \sum_{y<n \leq T} \frac{d(n)}{n^{3 / 4}} e(2 \sqrt{n x})\right|^{2} d x \\
& \ll T^{1+\varepsilon}+T^{3 / 2} \sum_{y<n \leq T} \frac{d^{2}(n)}{n^{3 / 2}} \\
&+T \sum_{y<m<n \leq T} \frac{d(n) d(m)}{(m n)^{3 / 4}(\sqrt{n}-\sqrt{m})} \\
& \ll T^{1+\varepsilon}+\frac{T^{3 / 2} \log ^{3} T}{y^{1 / 2}} \ll \frac{T^{3 / 2} \log ^{3} T}{y^{1 / 2}}
\end{aligned}
$$

where we used the estimates

$$
\sum_{n \leq u} d^{2}(n) \ll u \log ^{3} u, \quad \sum_{y<m<n \leq T} \frac{d(n) d(m)}{(m n)^{3 / 4}(\sqrt{n}-\sqrt{m})} \ll T^{\varepsilon} .
$$

Now suppose $y$ satisfies $y^{2 b\left(K_{0}\right)} \leq T$. Hence from Lemma 4.1 we get

$$
\int_{T}^{2 T}\left|\mathcal{R}_{1}\right|^{K_{0}} d x \ll T^{1+K_{0} / 4+\varepsilon}
$$


which implies

$$
\int_{T}^{2 T}\left|\mathcal{R}_{1}\right|^{A_{0}} d x \ll T^{1+A_{0} / 4+\varepsilon}
$$

since $A_{0} \leq K_{0}$. From (1.10) and (4.10) we get

$$
\int_{T}^{2 T}\left|\mathcal{R}_{2}\right|^{A_{0}} d x \ll \int_{T}^{2 T}\left(|\Delta(x)|^{A_{0}}+\left|\mathcal{R}_{1}\right|^{A_{0}}\right) d x \ll T^{1+A_{0} / 4+\varepsilon} .
$$

For any $2<A<A_{0}$, from (4.9), (4.11) and Hölder's inequality we get

$$
\begin{aligned}
\int_{T}^{2 T}\left|\mathcal{R}_{2}\right|^{A} d x & =\int_{T}^{2 T}\left|\mathcal{R}_{2}\right|^{\frac{2\left(A_{0}-A\right)}{A_{0}-2}+\frac{A_{0}(A-2)}{A_{0}-2}} d x \\
& \ll\left(\int_{T}^{2 T} \mathcal{R}_{2}^{2} d x\right)^{\frac{A_{0}-A}{A_{0}-2}}\left(\int_{T}^{2 T}\left|\mathcal{R}_{2}\right|^{A_{0}} d x\right)^{\frac{A-2}{A_{0}-2}} \\
& \ll T^{1+A / 4+\varepsilon} y^{-\frac{A_{0}-A}{2\left(A_{0}-2\right)}} .
\end{aligned}
$$

Thus, we have proved the following

Lemma 4.2. Suppose $T^{\varepsilon} \leq y \leq T^{1 / 2 b\left(K_{0}\right)}, 2<A<A_{0}$. Then

$$
\int_{T}^{2 T}\left|\mathcal{R}_{2}\right|^{A} d x \ll T^{1+A / 4+\varepsilon} y^{-\left(A_{0}-A\right) / 2\left(A_{0}-2\right)} .
$$

4.3. Proof of Theorem 1. Suppose $3 \leq k \leq K\left(A_{0}\right)$ and $T^{\varepsilon} \leq y \leq$ $T^{1 / 2 b\left(K_{0}\right)}$. By the elementary formula $(a+b)^{k}-\bar{a}^{k} \ll\left|a^{k-1} b\right|+|b|^{k}$, we get

$$
\int_{T}^{2 T} \Delta^{k}(x) d x=\int_{T}^{2 T} \mathcal{R}_{1}^{k} d x+O\left(\int_{T}^{2 T}\left|\mathcal{R}_{1}^{k-1} \mathcal{R}_{2}\right| d x\right)+O\left(\int_{T}^{2 T}\left|\mathcal{R}_{2}\right|^{k} d x\right) .
$$

If $k-1<A_{0} / 2$, then from (4.9), (4.10) and Cauchy's inequality we get $\int_{T}^{2 T}\left|\mathcal{R}_{1}^{k-1} \mathcal{R}_{2}\right| d x \ll\left(\int_{T}^{2 T}\left|\mathcal{R}_{1}\right|^{2(k-1)} d x\right)^{1 / 2}\left(\int_{T}^{2 T}\left|\mathcal{R}_{2}\right|^{2} d x\right)^{1 / 2} \ll T^{1+k / 4+\varepsilon} y^{-1 / 4}$. If $k-1 \geq A_{0} / 2$, then from (4.10), Lemma 4.2 and Hölder's inequality we get

$$
\begin{aligned}
\int_{T}^{2 T}\left|\mathcal{R}_{1}^{k-1} \mathcal{R}_{2}\right| d x & \ll\left(\int_{T}^{2 T}\left|\mathcal{R}_{1}\right|^{A_{0}} d x\right)^{(k-1) / A_{0}}\left(\int_{T}^{2 T}\left|\mathcal{R}_{2}\right|^{A_{0} /\left(A_{0}-k+1\right)} d x\right)^{\left(A_{0}-k+1\right) / A_{0}} \\
& \ll T^{1+k / 4+\varepsilon} y^{-\left(A_{0}-k\right) / 2\left(A_{0}-2\right)}
\end{aligned}
$$


Thus we have

$$
\int_{T}^{2 T}\left|\mathcal{R}_{1}^{k-1} \mathcal{R}_{2}\right| d x+\int_{T}^{2 T}\left|\mathcal{R}_{2}\right|^{k} d x \ll T^{1+k / 4+\varepsilon} y^{-\sigma\left(k, A_{0}\right)},
$$

where $\sigma\left(k, A_{0}\right)$ was defined in Section 1.1.

From (4.14) and (4.15) we get

$$
\int_{T}^{2 T} \Delta^{k}(x) d x=\int_{T}^{2 T} \mathcal{R}_{1}^{k} d x+O\left(T^{1+k / 4+\varepsilon} y^{-\sigma\left(k, A_{0}\right)}\right) .
$$

Now take $y=T^{1 / 2 b\left(K_{0}\right)}$. From Lemma 4.1 and (4.16) we get

$$
\begin{aligned}
\int_{T}^{2 T} \Delta^{k}(x) d x & =\frac{B_{k}(d)}{(\sqrt{2} \pi)^{k} 2^{k-1}} \int_{T}^{2 T} x^{k / 4} d x+O\left(T^{1+k / 4-\sigma\left(k, A_{0}\right) / 2 b\left(K_{0}\right)+\varepsilon}\right) \\
& =\frac{B_{k}(d)}{(\sqrt{2} \pi)^{k} 2^{k-1}} \int_{T}^{2 T} x^{k / 4} d x+O\left(T^{1+k / 4-\delta_{1}\left(k, A_{0}\right)+\varepsilon}\right) .
\end{aligned}
$$

Theorem 1 follows from (4.17) immediately.

4.4. Proof of Theorem 2. Suppose $T^{\varepsilon} \leq y \leq T^{1 / 3}$. By the truncated Voronoï formula (4.8), we have

$$
\mathcal{R}_{2}=(\sqrt{2} \pi)^{-1} x^{1 / 4} \sum_{y<n \leq N} \frac{d(n)}{n^{3 / 4}} \cos (4 \pi \sqrt{n x}-\pi / 4)+O\left(x^{1 / 2+\varepsilon} N^{-1 / 2}\right),
$$

where $y<N \ll T$. Using Ivić's large-value technique directly to $\mathcal{R}_{2}$ without modifications, we get the estimate

$$
\int_{T}^{2 T}\left|\mathcal{R}_{2}\right|^{A_{0}} d x \ll T^{1+A_{0} / 4+\varepsilon}
$$

with $A_{0}=184 / 19, T^{\varepsilon} \leq y \leq T^{1 / 3}$. We omit the details since the argument is completely the same as that of Ivić. Combining (4.18) and (1.10) we get

$$
\int_{T}^{2 T}\left|\mathcal{R}_{1}\right|^{A_{0}} d x \ll T^{1+A_{0} / 4+\varepsilon}
$$

with $A_{0}=184 / 19, T^{\varepsilon} \leq y \leq T^{1 / 3}$.

By the same argument as in the last subsection, we deduce that for $T^{\varepsilon} \leq y \leq T^{1 / 3}$

$$
\int_{T}^{2 T} \Delta^{k}(x) d x=\int_{T}^{2 T} \mathcal{R}_{1}^{k} d x+O\left(T^{1+k / 4+\varepsilon} y^{-\sigma(k, 184 / 19)}\right) .
$$


Take $y=T^{1 /(2 b(k)+2 \sigma(k, 184 / 19))}$. From Lemma 4.1 again we get

$$
\begin{aligned}
\int_{T}^{2 T} \Delta^{k}(x) d x & =\frac{B_{k}(d)}{(\sqrt{2} \pi)^{k} 2^{k-1}} \int_{T}^{2 T} x^{k / 4} d x+O\left(T^{1+k / 4-\frac{\sigma(k, 184 / 19)}{2 b(k)+2 \sigma(k, 184 / 19)}+\varepsilon}\right) \\
& =\frac{B_{k}(d)}{(\sqrt{2} \pi)^{k} 2^{k-1}} \int_{T}^{2 T} x^{k / 4} d x+O\left(T^{1+k / 4-\delta_{2}(k, 184 / 19)+\varepsilon}\right)
\end{aligned}
$$

and Theorem 2 follows.

5. Proofs of other theorems. $P(x)$ has the following truncated Voronoï formula:

$$
P(x)=-\frac{1}{\pi} \sum_{n \leq N} r(n) n^{-3 / 4} x^{1 / 4} \cos (4 \pi \sqrt{n x}+\pi / 4)+O\left(x^{1 / 2+\varepsilon} N^{-1 / 2}\right)
$$

for $1 \leq N \ll x$, which follows from Lemma 3 of Müller [16]. Moreover, $A(x)$ has the following truncated Voronoï formula:

$$
\begin{aligned}
A(x)= & \frac{1}{\pi \sqrt{2}} x^{\kappa / 2-1 / 4} \sum_{n \leq N} a(n) n^{-\kappa / 2-1 / 4} \cos (4 \pi \sqrt{n x}-\pi / 4) \\
& +O\left(x^{\kappa / 2+\varepsilon} N^{-1 / 2}\right)
\end{aligned}
$$

for $1 \leq N \ll x$, which is a special case of Theorem 1.1 of Jutila [13]. So in the same way as in the last section, we get Theorems 3 and 4 .

Now we prove Theorem 5. We shall follow Ivić [10]. Define

$$
\Delta^{*}(x):=\frac{1}{2} \sum_{n \leq 4 x}(-1)^{n} d(n)-x(\log x+2 \gamma-1), \quad x>0 .
$$

Jutila [12] proved that

$$
\int_{0}^{T}\left(E(t)-2 \pi \Delta^{*}\left(\frac{t}{2 \pi}\right)\right)^{2} d t \ll T^{4 / 3} \log ^{3} T
$$

which means that $E(t)$ is well approximated by $2 \pi \Delta^{*}(t / 2 \pi)$ at least in the mean square sense.

Suppose $A_{0}>9$ is a real number such that both (1.10) and (1.32) hold. Since (see Jutila [11])

$$
\Delta^{*}(x)=-\Delta(x)+2 \Delta(2 x)-\frac{1}{2} \Delta(4 x),
$$

from (1.10) we get

$$
\int_{0}^{T}\left|\Delta^{*}(t)\right|^{A_{0}} d t \ll T^{1+A_{0} / 4+\varepsilon}
$$


Then from (1.32), (5.3), (5.4) and Hölder's inequality, for any $3 \leq k<A_{0}$ we get

$$
\begin{aligned}
\int_{0}^{T} E^{k}(t) & d t-(2 \pi)^{k+1} \int_{0}^{T / 2 \pi}\left(\Delta^{*}(t)\right)^{k} d t \\
& =\int_{0}^{T}\left(E^{k}(t)-\left(2 \pi \Delta^{*}\left(\frac{t}{2 \pi}\right)\right)^{k}\right) d t \\
& \ll \int_{0}^{T}\left|E(t)-2 \pi \Delta^{*}\left(\frac{t}{2 \pi}\right)\right|\left(|E(t)|^{k-1}+\left|\Delta^{*}\left(\frac{t}{2 \pi}\right)\right|^{k-1}\right) d t \\
& \ll T^{1+k / 4-\sigma\left(k, A_{0}\right) / 3+\varepsilon},
\end{aligned}
$$

where $\sigma\left(k, A_{0}\right)$ was defined in Section 1.1. By (5.5) the problem is reduced to evaluating the integral $\int_{0}^{T}\left(\Delta^{*}(t)\right)^{k} d t$. For $1 \ll N \ll x$, we have $[10,(7)]$

$$
\begin{aligned}
\Delta^{*}(x)= & \frac{1}{\pi \sqrt{2}} \sum_{n \leq N}(-1)^{n} d(n) n^{-3 / 4} x^{1 / 4} \cos (4 \pi \sqrt{n x}-\pi / 4) \\
& +O\left(x^{1 / 2+\varepsilon} N^{-1 / 2}\right),
\end{aligned}
$$

which is similar to (4.8). Let $d^{*}(n)=(-1)^{n} d(n)$. Then in the same way as in the proof of Theorem 1, we get the asymptotic formula

$$
\int_{1}^{T}\left(\Delta^{*}(t)\right)^{k} d t=\frac{B_{k}\left(d^{*}\right)}{(1+k / 4) 2^{3 k / 2-1} \pi^{k}} T^{1+k / 4}+O\left(T^{1+k / 4-\delta_{1}\left(k, A_{0}\right)+\varepsilon}\right)
$$

for any $3 \leq k<A_{0}$.

We shall use

Lemma 5.1. Suppose $1 \leq l<k$ are fixed integers and $\left(n_{1}, \ldots, n_{k}\right) \in \mathbb{N}^{k}$. If

$$
\sqrt{n_{1}}+\ldots+\sqrt{n_{l}}=\sqrt{n_{l+1}}+\ldots+\sqrt{n_{k}}
$$

then $2 \mid\left(n_{1}+\ldots+n_{k}\right)$.

Proof. For any $n \in \mathbb{N}$, let $h(n)$ denote the squarefree part of $n$. Let $\mathcal{S}=\left\{h\left(n_{1}\right), \ldots, h\left(n_{k}\right)\right\} \cap \mathbb{N}$ and $s=\# \mathcal{S}$. For convenience, write

$$
\mathcal{S}=\left\{h_{1}, \ldots, h_{s}\right\}, \quad I=\{1, \ldots, l\}, \quad J=\{l+1, \ldots, k\} .
$$

From Lemma 2.1 we can write $I=\bigcup_{e=1}^{s} I_{e}, J=\bigcup_{e=1}^{s} J_{e}$ so that for each $1 \leq e \leq s$

$$
\sum_{i \in I_{e}} \sqrt{n_{i}}=\sum_{j \in J_{e}} \sqrt{n_{j}}
$$

and all $n_{i}\left(i \in I_{e}\right)$ and $n_{j}\left(j \in J_{e}\right)$ have the same squarefree part $h_{e}$. Namely 
we have $(1 \leq e \leq s)$

$$
n_{i}=m_{i}^{2} h_{e}\left(i \in I_{e}\right), \quad n_{j}=m_{j}^{2} h_{e}\left(j \in I_{e}\right), \quad \sum_{i \in I_{e}} m_{i}=\sum_{j \in J_{e}} m_{j} .
$$

Thus we get

$$
\begin{aligned}
n_{1}+\ldots+n_{k} & =\sum_{e=1}^{s}\left(\sum_{i \in I_{e}} n_{i}+\sum_{j \in J_{e}} n_{j}\right) \\
& =\sum_{e=1}^{s}\left(\sum_{i \in I_{e}} m_{i}^{2} h_{e}+\sum_{j \in J_{e}} m_{j}^{2} h_{e}\right) \equiv \sum_{e=1}^{s}\left(\sum_{i \in I_{e}} m_{i}+\sum_{j \in J_{e}} m_{j}\right) h_{e} \\
& =2 \sum_{e=1}^{s} h_{e} \sum_{i \in I_{e}} m_{i} \equiv 0(\bmod 2)
\end{aligned}
$$

where we used the simple congruence $n^{2} \equiv n(\bmod 2)$.

From Lemma 5.1, for any $1 \leq l<k$ we get

$$
\begin{aligned}
s_{k ; l}\left(d^{*}\right)= & \sum_{\sqrt{n_{1}}+\ldots+\sqrt{n_{l}}=\sqrt{n_{l+1}}+\ldots+\sqrt{n_{k}}}(-1)^{n_{1}+\ldots+n_{k}} \frac{d\left(n_{1}\right) \ldots d\left(n_{k}\right)}{\left(n_{1} \ldots n_{k}\right)^{3 / 4}} \\
= & \sum_{\sqrt{n_{1}}+\ldots+\sqrt{n_{l}}=\sqrt{n_{l+1}}+\ldots+\sqrt{n_{k}}} \frac{d\left(n_{1}\right) \ldots d\left(n_{k}\right)}{\left(n_{1} \ldots n_{k}\right)^{3 / 4}}=s_{k ; l}(d) .
\end{aligned}
$$

Hence we conclude that

$$
B_{k}\left(d^{*}\right)=B_{k}(d)
$$

From (5.5), (5.7) and (5.8) we get (1.33).

Similarly to Theorem 2, we can prove the asymptotic formula

$$
\int_{1}^{T}\left(\Delta^{*}(t)\right)^{k} d t=\frac{B_{k}(d)}{(1+k / 4) 2^{3 k / 2-1} \pi^{k}} T^{1+k / 4}+O\left(T^{1+k / 4-\delta_{2}(k, 576 / 61)+\varepsilon}\right)
$$

for any $3 \leq k \leq 9$, which combined with (5.5) yields the second part of Theorem 3.

Note added in proof. Recently M. N. Huxley, Exponential sums and lattice points III, Proc. London Math. Soc. 87 (2003), 591-609, proved

$$
\Delta(x) \ll x^{131 / 416}(\log x)^{26957 / 8320},
$$

which implies that the exponent 184/19 for which the formula (1.10) holds can be improved to $A_{0}=262 / 27$. Correspondingly, the exponent $\delta_{2}(k, 184 / 19)$ in Theorem 2 can be improved to $\delta_{2}(k, 262 / 27)$ for $k=6,7,8,9$. The author deeply thanks Professor A. Schinzel for informing him about M. N. Huxley's new result. 


\section{References}

[1] F. V. Atkinson, The mean value of the Riemann zeta-function, Acta Math. 81 (1949), 353-376.

[2] A. S. Besicovitch, On the linear independence of fractional powers of integers, J. London Math. Soc. 15 (1940), 3-6.

[3] Y. C. Cai, On the third and fourth power moments of Fourier coefficients of cusp forms, Acta Math. Sinica (N.S.) 13 (1997), 443-452.

[4] P. Deligne, La conjecture de Weil I, Inst. Hautes Études Sci. Publ. Math. 43 (1974), 273-307.

[5] D. R. Heath-Brown, The distribution and moments of the error term in the Dirichlet divisor problem, Acta Arith. 60 (1992), 389-415.

[6] M. N. Huxley, Area, Lattice Points, and Exponential Sums, London Math. Soc. Monogr. (N.S.) 13, Oxford Univ. Press, 1996.

[7] A. Ivić, The Riemann Zeta-Function, Wiley, 1985.

[8] —, Lectures on Mean Values of the Riemann Zeta-Function, Tata Inst. Fund. Res. Lectures on Math. and Phys. 82, Bombay, 1991.

[9] —, Large values of certain number-theoretic error terms, Acta Arith. 56 (1990), $135-159$.

[10] - On some problems involving the mean square of $\zeta\left(\frac{1}{2}+i t\right)$, Bull. Cl. Sci. Math. Nat. Sci. Math. 23 (1998), 71-76.

[11] M. Jutila, Riemann's zeta-function and the divisor problem, I, II, Ark. Mat. 21 (1983), 75-96 and 31 (1993), 61-70.

[12] —, On a formula of Atkinson, in: Topics in Classical Number Theory, Vol. I, II (Budapest, 1981), Colloq. Math. Soc. János Bolyai 34, North-Holland, Amsterdam, 1984, 807-823.

[13] - Lectures on a Method in the Theory of Exponential Sums, Tata Inst. Fund. Res. Lectures on Math. and Phys. 80, Bombay, 1987.

[14] I. Kátai, The number of lattice points in a circle, Ann. Univ. Sci. Budapest. Eötvös Sect. Math. 8 (1965), 39-60 (in Russian).

[15] T. Meurman, On the mean square of the Riemann zeta-function, Quart. J. Math. Oxford Ser. (2) 38 (1987), 337-343.

[16] W. Müller, On the asymptotic behaviour of the ideal counting function in quadratic number fields, Monatsh. Math. 108 (1989), 301-323.

[17] K. C. Tong, On divisor problem III, Acta Math. Sinica 6 (1956), 515-541.

[18] K. M. Tsang, Higher-power moments of $\Delta(x), E(t)$ and $P(x)$, Proc. London Math. Soc. (3) 65 (1992), 65-84.

[19] G. Voronoï, Sur une fonction transcendante et ses applications à la sommation de quelques séries, Ann. Sci. École Norm. Sup. (3) 21 (1904), 207-267, 459-533.

[20] W. G. Zhai, On higher-power moments of $\Delta(x)$, Acta Arith. 112 (2004), 367-395.

Department of Mathematics

Shandong Normal University

Jinan, Shandong 250014, P.R. China

E-mail: zhaiwg@hotmail.com 